\title{
Phylogenetic analysis of Notalina (Neonotalina) Holzenthal (Trichoptera: Leptoceridae), with the description of two new species from southeastern Brazil
}

\author{
ADOLFO R. CALOR ${ }^{1}$, RALPH W. HOLZENTHAL ${ }^{2}$ \& DALTON S. AMORIM ${ }^{1}$ \\ ${ }^{1}$ Departamento de Biologia, Faculdade de Filosofia, Ciências e Letras de Ribeirão Preto, Universidade de \\ São Paulo. Av. Bandeirantes, 3900, CEP 14040-901, Ribeirão Preto, São Paulo, Brazil (acalor@usp.br, \\ dsamorim@usp.br) \\ ${ }^{2}$ Department of Entomology, University of Minnesota, 219 Hodson Hall, 1980 Folwell Avenue, St. Paul, MN \\ 55108,USA (holze001@umn.edu)
}

\begin{abstract}
Two new species of Notalina (Neonotalina) Holzenthal 1986 from southeastern Brazil are described and illustrated, N. (Neonotalina) froehlichi Calor \& Holzenthal and N. (Neonotalina) paulista Calor \& Holzenthal. Phylogenetic analyses confirm the placement of the nine Neotropical species of Notalina into two species groups, the roraima species-group and the brasiliana speciesgroup, with northern South American and southeastern Brazilian patterns of distribution, respectively. Additional collection records of previously described species from Brazil are provided.
\end{abstract}

Key words: Trichoptera, Leptoceridae, Notalina, Neotropical, systematics, phylogeny

\section{Introduction}

The Leptoceridae Leach 1815, or long-horned caddisflies, are recognized by their long, narrow forewings and very long, filiform antennae (Neboiss 1977, Morse \& Holzenthal 1987). The family is cosmopolitan and contains 1567 described species, making it the second largest family in the order after the Hydroptilidae (1677 species) in species diversity (Morse 2003). Like the Hydroptilidae, long-horned caddisflies are especially diverse in warmer regions. There are 148 species in the Neotropics (Flint et al. 1999), 41 of them in Brazil (Paprocki et al. 2004). Species are found in an extensive variety of habitats, ranging from high mountain torrents and cascades to meandering lowland rivers, 
sloughs, and even semi-terrestrial habitats (Holzenthal 1988). Leptocerid larvae construct tubular cases from a wide range of materials, including, at least in the Neotropics, small sand grains, pieces of grass stems, leaf detritus, small hollowed-out twigs, or entirely from silk (Holzenthal 1988). Larval feeding habits are also varied and include predators, shredders, scrapers, and some that feed on living plants.

\section{Systematics}

The family Leptoceridae comprises two subfamilies, Leptocerinae Leach 1815 and Triplectidinae Ulmer 1906 (Morse 1981). The Leptocerinae are cosmopolitan, and the Triplectidinae are distributed in the Australian and Neotropical regions (Morse 1981, Holzenthal 1986a, Morse \& Holzenthal 1987), with a putative member in southern Africa (Moor 1997). Monophyly of Triplectidinae is based on two characters, the loss of the primitive phallic parameres and marked reduction of the apical phallicata, while monophyly of Leptocerinae is supported by loss of one branch of the median vein (third cell) in the hind wing, loss of the sectoral crossvein in the hind wing, and by reduction of the adult tibial spur formula from 2,4,4 to 2,2,4 (Morse 1981).

Triplectidinae contains the tribes Triplectidini, Hudsonemini and Grumichellini (Morse 1981, Holzenthal 1986b, Morse \& Holzenthal 1987). Monophyly of Hudsonemini is supported by the male phallotremal sclerite developed into a pair of broad, vertical plates (Morse 1981, Holzenthal 1986b), the presence of secondary setae on the lateral sclerite of each anal proleg of the larva, and the absence (or fusion of vestige) of the 2 nd article of the inferior appendage of the male (Holzenthal 1986b). The phylogeny of the genera of Hudsonemini, Notalina Mosely 1936, Hudsonema Mosely 1936, and Condocerus Neboiss 1977, was inferred by Holzenthal (1986b), who proposed a sistergroup relationship between Hudsonema and Notalina.

Mosely (1936), in his revision of the Triplectidinae, erected the genus Notalina for three Australian species, Notalina parkeri (type species), N. delicatula (Ulmer) 1907, and N. flava (Ulmer) 1907. Since then, additional Australian species have been described, making Notalina one of the most diverse triplectidine genera in the region (Neboiss 1986). Fifteen species are now known from the Australian fauna, including the following additional species: N. arena St. Clair 1991, N. bifaria Neboiss 1977, N. dwellinga Neboiss 1982, N. fulva Kimmins 1953, N. gungara Neboiss 1984, N. moselyi Kimmins 1953, N. nigra (Mosely) 1953, N. ordina St. Clair 1991, N. pseudodelicatula Neboiss 1982, N. salina St. Clair 1991, N. spira St. Clair 1991, and N. tillyardi Kimmins 1953. Mosely erected the genus Notalina with a single character, "absence of fork one in the hindwings." This character is dubious because in a larger series of specimens of $N$. nigra, fork one in the hind wing sometimes is indistinct or occasionally present (Neboiss 1977). Holzenthal (1986b) proposed that the synapomorphy for Notalina was the very narrow fork 1 of the hind wing, with a tendency to become lost. 
In addition, Holzenthal (1986a) described seven new species from South America and placed them in a new subgenus, Neonotalina. He further placed the South American species in two species groups based on characteristics of the male genitalia, the brasiliana group from southeastern Brazil (N. brasiliana Holzenthal 1986, N. morsei Holzenthal 1986, N. cipo Holzenthal 1986, N. hamiltoni Holzenthal 1986), and the roraima group from Venezuela, Peru, and Colombia (N. roraima Holzenthal 1986, N. nanay Holzenthal 1986, N. matthiasi Holzenthal 1986). Below, we infer the phylogenetic relationships among the seven described species and two new species of Notalina (Neonotalina) and discuss biogeographic implications.

\section{Materials and methods}

Material examined was borrowed from, or will be deposited in, the following institutions: Museu de Zoologia da Universidade de São Paulo, Brazil (MZUSP), Coleção Entomológica, Departamento de Zoologia, Instituto de Biologia, Universidade Federal do Rio de Janeiro, Brazil (UFRJ), University of Minnesota Insect Collection, St. Paul, Minnesota, USA (UMSP), and National Museum of Natural History, Smithsonian Institution, Washington, DC, USA (NMNH). Methods used in the collection, preparation, examination, illustration, and description of the species were those discussed by Holzenthal and Andersen (2004) and Blahnik and Holzenthal (2004). The morphological terminology used in this paper follows that described and figured by Schmid (1980) and implemented by Holzenthal (1986a).

The phylogenetic analysis was carried out in PAUP* $4.0 \mathrm{~b} 10$ (Swofford 2001) on a Power Macintosh G5. The Branch-and-Bound search routine (Hendy \& Penny 1982) was performed to obtain the optimal tree(s). All characters were treated as unordered and equally weighted in the initial analysis.

Even though differential weighting of characters remains a point of controversy (Hillis 1991, Kluge 1997), analyses using successive weighting (Farris 1969) and implied weighting (Goloboff 1993, 1995) were employed for comparative purposes and to search for other hypotheses of character evolution, in short, to examine the consistency of the results under more than one weighting approach (Carpenter et al. 2000). The successive weighting analyses were based on either rescaled consistency indices (RC) or retention indices (RI), and were also carried out using PAUP*. Methods for a posteriori character weighting, such as successive weighting, are generally used to find topologies supported by the most consistent characters, thus facilitating the choice of a preferred tree from a initial set obtained from equally weighted analyses (Carpenter 1988, 1994; Carpenter et al. 1993). Nevertheless, in the present context, a posteriori weighting was undertaken to assess the effects of weighting against homoplastic characters, irrespective of the number of trees obtained with equally weighted analysis. 
The ACCTRAN option was used for optimization of states of characters. The missing data and inapplicable data were treated as (?) and (-), respectively, despite the fact that PAUP* treats these in the same manner. Nevertheless, if a matrix is considered a description of taxa, this information contributes to its completeness and future utility.

The seven described Notalina (Neonotalina) and the two new species represent the putative ingroup, its monophyly tested by outgroup analysis (Nixon and Carpenter 1993). Outgroup species included in the analysis were Notalina (Notalina) (seven species), Hudsonema flaminii (Navás) 1926, Condocerus aptus Neboiss 1982, Grumichella aequiunguis Flint 1983, Atanatolica brasiliana (Brauer) 1865, and Triplectides gracilis (Burmeister) 1839.

We scored 23 binary and eight multistate characters. Female, larval and pupal characters are unknown for most of species, and were excluded from the analysis. The characters used in our analysis are those of the male genitalia, tibial spurs, and wing venation (Table 1). The character matrix (Table 2) was constructed using NDE (Page 2001).

TABLE 1. Characters and states used in the phylogenetic analyses.

1. Width of preanal appendage: (0) narrow; (1) intermediate; (2) wide.

2. Shape of preanal appendage: (0) club shaped; (1) tubular.

3. Shape of apex of preanal appendage: (0) rounded; (1) acuminate; (2) truncate.

4. Number of terminal processes of segment $X:(\mathbf{0})$ one; (1) two; (2) four.

5. Length of terminal processes of segment $X$ : (0) short; (1) intermediate; (2) long.

6. Dorsal margin of segment X: (0) straight (without median protuberance); (1) convex (with median protuberance).

7. Length of inferior appendage: (0) short; (1) long; (2) very long.

8. Second article of inferior appendage: (0) present; (1) absent.

9. Basodorsal process of inferior appendage: (0) absent; (1) present.

10. Length of basodorsal process of inferior appendage: (0) short; (1) long.

11. Basomesal process of inferior appendage: (0) absent; (1) present.

12. Direction of apical region of basomesal process of inferior appendage: (0) directed dorsad; (1) directed ventrad; (2) directed posteriorly.

13. Phallic apodeme: (0) well developed; (1) underdeveloped.

14. Lateral processes of phallobase: (0) absent; (1) present.

15. Shape of lateral processes of phallobase apically: (0) acuminate; (1) rounded.

16. Direction of lateral processes of phallobase (ventral view): (0) directed ventrally; (1) directed dorsally; (2) directed posteriorly.

17. Direction of lateral processes of phallobase apically (dorsal view): (0) laterally curved; (1) straight.

18. Size of phallotremal sclerite: (0) small; (1) large.

19. Phallotremal sclerite apex (U-shaped): (0) apices approximate; (1) apices separate.

20. Dorsobasal process of phallobase: (0) absent; (1) present.

21. Forewing vein $R 2+3$ length (fork 1 petiolate): (0) short; (1) long. 
22. Forewing crossvein Sc-Rl: (0) more distal; (1) less distal.

23. Forewing crossveins $(R 2+3-R 4 ; R 3-M 1+2 ; M-C u 1 a)$ : (0) not aligned; (1) aligned.

24. Forewing vein $M-C u$ length (thiridial cell petiolate): (0) short; (1) long.

25. Hind wing vein SR length: (0) very short; (1) short; (2) long.

26. Hind wing crossveins $R 2+3-R 4$ and $R 4-M 1+2$ : (0) not aligned; (1) aligned.

27. Hind wing fork 1: (0) absent; (1) present.

28. Hind wing fork 1 width: (0) narrow; (1) wide.

29. Hind wing vein R1: (0) absent; (1) present.

30. Number of foretibial spurs: (0) zero; (1) two.

31. Number of hindtibial spurs: (0) two; (1) four.

TABLE 2. Data matrix. Characters as listed in Table 1.

\begin{tabular}{|c|c|c|c|c|c|c|c|c|c|c|c|c|c|c|c|c|c|c|c|c|c|c|c|c|c|c|c|c|c|c|c|}
\hline . $g r c$ & & & & 1 & 2 & 0 & 1 & 0 & & & 1 & & & 0 & & & & & & 0 & 0 & & () & 0 & & & & 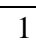 & & & \\
\hline$f_{r}$ & 1 & 0 & 0 & 2 & 1 & 1 & 2 & 1 & 1 & 1 & 1 & 0 & 0 & 1 & 0 & & & & & 1 & 1 & 0 & 1 & 0 & 1 & 1 & & 0 & 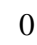 & 1 & 1 \\
\hline$p$ & 1 & & 0 & 1 & 2 & 1 & 2 & 1 & 1 & 1 & 1 & 0 & 0 & 1 & 0 & & U & & & 0 & 1 & 0 & 1 & 0 & 1 & & & 0 & 0 & 1 & \\
\hline$b r$ & 0 & 1 & 0 & 2 & 1 & 0 & 1 & 1 & 1 & 1 & 1 & 0 & 0 & 1 & 0 & & 0 & & & 1 & 1 & 0 & 1 & 0 & 1 & 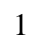 & & 0 & 0 & 1 & 1 \\
\hline N. cipo & 0 & 1 & 0 & 1 & 1 & 1 & 1 & 1 & 1 & 1 & 1 & 0 & 0 & 1 & 0 & & 0 & & & 0 & 1 & 0 & 1 & 0 & 1 & & & 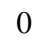 & 0 & 1 & \\
\hline N. $m$ & 1 & 0 & 2 & 1 & 1 & 1 & 1 & 1 & 1 & 1 & 1 & 0 & 0 & 1 & 0 & 1 & & & & 0 & 1 & 0 & 1 & 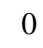 & & & & o & & 1 & 1 \\
\hline$h c$ & 1 & 0 & 0 & 1 & 1 & 0 & 2 & 1 & 1 & 1 & 1 & 1 & 0 & 1 & 0 & 1 & & & & 0 & 1 & 0 & 1 & 0 & 1 & 1 & & 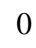 & 0 & 1 & 1 \\
\hline $\mathrm{v} . m$ & 2 & 0 & 1 & 1 & 1 & 1 & 1 & 1 & 1 & 1 & 1 & 2 & 1 & 1 & 1 & 2 & 1 & & & 0 & 1 & 0 & 1 & 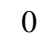 & 1 & 1 & & 0 & 0 & 1 & 1 \\
\hline$n$ & 1 & 1 & 1 & 0 & 0 & 0 & 1 & 1 & 1 & 1 & 1 & 2 & 1 & 1 & 1 & 2 & & & & 0 & 1 & 0 & 1 & ( & & & & 0 & 0 & 1 & 1 \\
\hline N. ro & 1 & 0 & 1 & 1 & 0 & 0 & 2 & 1 & 1 & 1 & 1 & 0 & 1 & 1 & 1 & 2 & 1 & 0 & & 0 & 1 & 0 & 1 & 0 & 1 & & & 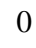 & & 1 & 1 \\
\hline V. $p$ & 0 & 1 & 1 & 1 & 2 & 0 & 1 & 1 & 1 & 0 & 0 & - & $?$ & 1 & 0 & 0 & $?$ & ? & & 0 & 1 & 0 & 0 & 1 & & 0 & & - & 1 & 1 & 1 \\
\hline N. $b i$ & 0 & 1 & 1 & 1 & 2 & 0 & 1 & 1 & 1 & 0 & 0 & - & $?$ & 1 & 0 & 0 & $?$ & $?$ & & ? & 1 & 1 & 0 & 1 & 0 & & & 0 & 1 & 1 & 1 \\
\hline N. delicatula & 0 & 1 & 1 & 1 & 2 & 0 & 1 & 1 & 1 & 0 & 0 & - & 1 & 1 & 0 & 0 & & 1 & 8 & 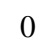 & 1 & 1 & 0 & 1 & 0 & & & 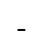 & 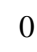 & 1 & 1 \\
\hline N. flava & 0 & 1 & 1 & 1 & 2 & 0 & 1 & 1 & 1 & 0 & 0 & - & 1 & 1 & 0 & 0 & & 1 & ? & 0 & 1 & 1 & 0 & 1 & & & & - & 1 & 1 & 1 \\
\hline$I$ & 1 & 0 & 0 & 1 & 2 & 0 & 1 & 1 & 1 & 0 & 0 & - & $?$ & 1 & 0 & 0 & & $?$ & ? & $?$ & 1 & 1 & 0 & 1 & & & & 0 & 1 & 1 & 1 \\
\hline N. ful & 0 & 1 & 1 & 1 & 2 & 0 & 1 & 1 & 1 & 0 & 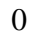 & - & 0 & 1 & 0 & 0 & & & & 0 & 1 & 1 & 0 & 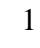 & & & & - & 1 & 1 & 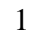 \\
\hline N. mo & 0 & 1 & 1 & 1 & 2 & 0 & 1 & 1 & 1 & 0 & 0 & - & $?$ & 1 & 0 & 0 & & & & $?$ & 1 & 1 & 0 & 1 & 0 & & & - & 1 & 1 & 1 \\
\hline 1. flaminii & 1 & 1 & 1 & 1 & 2 & 0 & 0 & 1 & 0 & 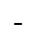 & 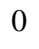 & - & 1 & 0 & 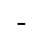 & 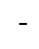 & & & & 0 & 1 & 1 & 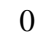 & 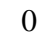 & & & & 1 & 1 & 1 & 1 \\
\hline C. aptus & 1 & 1 & 1 & 1 & 2 & 0 & 0 & 1 & 1 & 0 & 0 & - & $?$ & $?$ & $?$ & ? & & & & $?$ & 1 & 1 & 0 & 0 & & & & 1 & 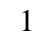 & 1 & 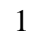 \\
\hline G. $a e$ & 2 & 0 & 1 & 1 & 1 & 0 & 2 & 0 & 0 & - & 0 & - & 1 & 0 & - & - & & & & 0 & 0 & 1 & 0 & 0 & & & & - & 1 & 0 & 0 \\
\hline 1. brasiliana & 1 & 0 & 1 & 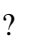 & 1 & 0 & 2 & 0 & 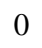 & & 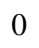 & & 1 & $\sigma$ & & & & & & 0 & 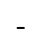 & 1 & 0 & & & & & & & & \\
\hline
\end{tabular}

\section{Taxonomy}

\section{Notalina froehlichi, Calor \& Holzenthal, new species}

Fig. 1

As in other members of brasiliana species-group, this species possesses lateral, acuminate flanges on the male phallobase and well-developed phallotremal sclerites. It has two pairs 
zootaxa of processes on abdominal segment $\mathrm{X}$ and the median region of this segment is thick. In 1131 addition, the mesodorsal process of the inferior appendage has its apex dorsally curved in lateral view.

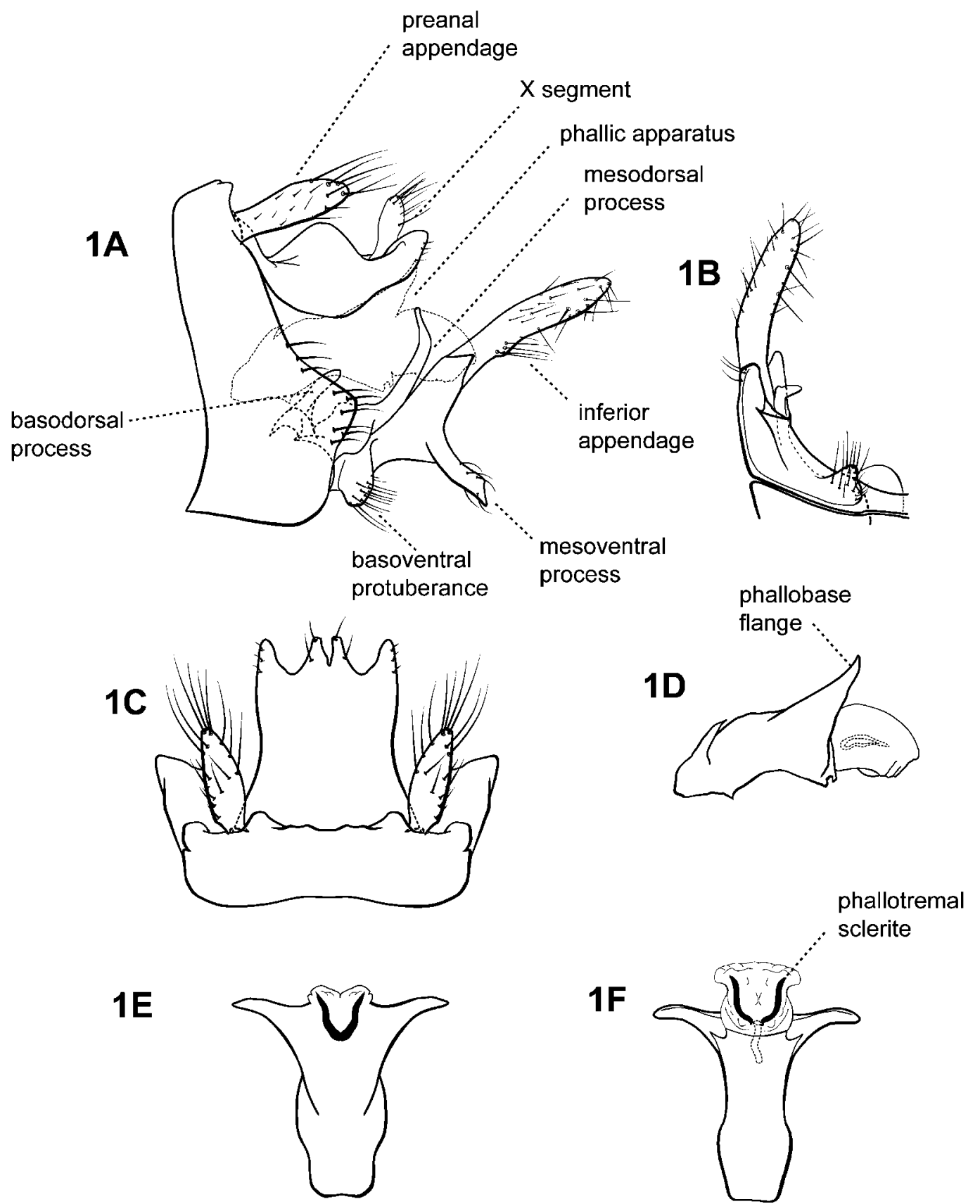

FIGURE 1. Notalina froehlichi, new species. Male genitalia: A-abdominal segments IX and X, lateral; B-inferior appendage, ventral; C-abdominal segments IX and X, dorsal; D-phallic apparatus, lateral; E-same, dorsal; F-same, ventral. 
Adult: Color brown, with small, white, wing spots. Forewing length $10 \mathrm{~mm}$.

Male: Genitalia as in Fig. 1. Abdominal segment IX broadest ventrolaterally, with pair of small, dorsal protuberances. Segment X elevated mesally in lateral view, bearing pair of subequal dorsomesal and ventrolateral processes apically, dorsomesal process claviform, rounded apically; ventrolateral process broadly digitate, rounded apically. Preanal appendage long, about half-length of segment X, digitate, setose. Inferior appendage with apical portion elongate, digitate, setose; basal portion bearing various processes: short, flat, apically rounded basodorsal process; long, slender mesodorsal process, its apex narrowed, dorsally directed in lateral view; very short, rounded, setose, basal protuberance; and long, digitate, apically pointed mesoventral process. Phallic apparatus with phallic apodeme and phallobase well developed; phallobase with pair of dorsolaterally directed, acuminate flanges; phallotremal sclerite well developed, roughly U-shaped in dorsal view.

Holotype male: BRAZIL: Minas Gerais: Rio Caraça, near Santa Barbara, $20^{\circ} 01^{\prime 22}$ "S, 043 ${ }^{\circ} 28^{\prime} 45^{\prime \prime} \mathrm{W}, 728$ m, 9.xi.2001 (Holzenthal, Amarante, Blahnik, \& Paprocki) (UMSP 000081361) (MZUSP).

Paratype: BRAZIL: Minas Gerais: Rio Caraça, near Santa Barbara, 2001'22"S, 04328'45"W, 728 m, 9.xi.2001 (Holzenthal, Amarante, Blahnik, \& Paprocki) 1 male (UMSP).

Etymology: This species name is dedicated to Dr. Claudio G. Froehlich, the notable Brazilian entomologist from FFCLRP/USP, who has been instrumental in contributing to our knowledge of Brazilian aquatic insects.

\section{Notalina paulista, Calor \& Holzenthal, new species}

Fig. 2

This species has a pair of long, terminal processes and a pair of small, median processes on abdominal segment $\mathrm{X}$. As in other members of the brasiliana species-group, it possesses lateral acuminate flanges on the male phallobase and well-developed phallotremal sclerites.

Adult: Color brown, with small white wing spots. Forewing length $9-10 \mathrm{~mm}$.

Male: Genitalia as in Fig. 2. Segment IX broadest ventrolaterally, with pair of small, closely situated dorsal protuberances. Segment X concave dorsally in lateral view, bearing pair of mesal dorsolateral and apical processes; dorsolateral process short, digitate, bearing many short fine setae; apical process well developed, long, acuminate, bearing fine apical setae. Preanal appendage long, about half-length of segment X, digitate, setose, apically rounded. Inferior appendage with apical portion very elongate, digitate, setose; basal portion bearing various processes: very short, flat, apically rounded basodorsal process; long, slender mesodorsal process; very short, rounded, setose, basal protuberance; and short, thumb-shaped, setose, mesoventral process. Phallic apparatus with phallic apodeme 

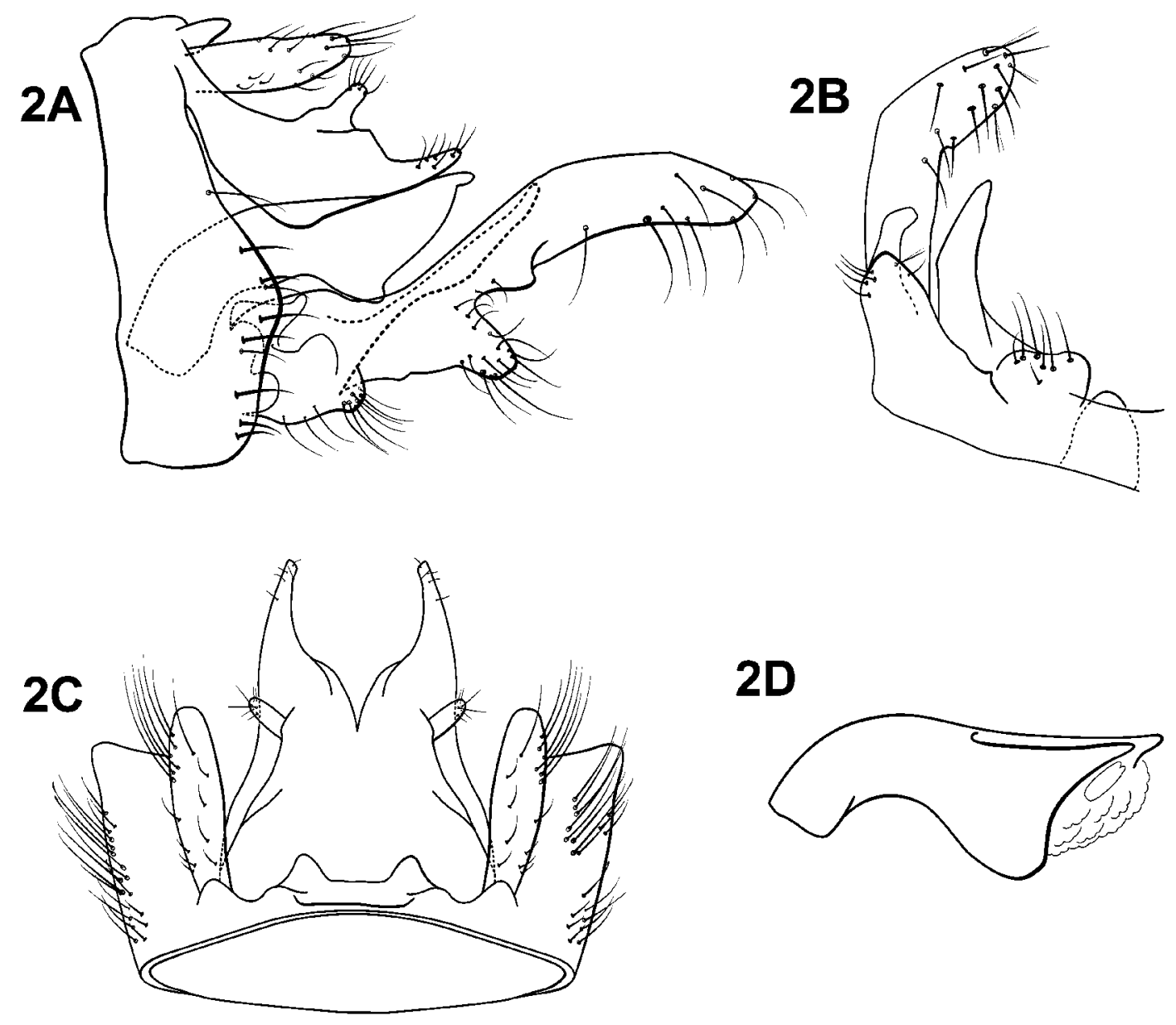

\section{D}
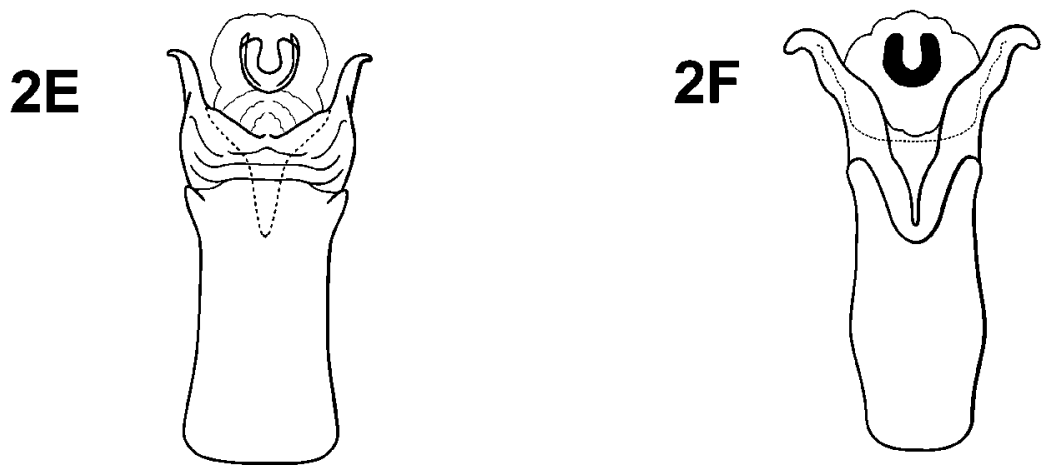

FIGURE 2. Notalina paulista, new species. Male genitalia: A-abdominal segments IX and X, lateral; B-inferior appendage, ventral; C-abdominal segments IX and X, dorsal; D-phallic apparatus, lateral; E-same, dorsal; F-same, ventral. 
Holotype male: BRAZIL: São Paulo: Cachoeira do Paredão, Lajeado, Serra da Bocaina, 22 43'32"S, 044³7'16"W, 1550 m, 1-2.iii.2002 (Holzenthal, Blahnik, Paprocki, \& Prather) (UMSP000200915) (MZUSP).

Paratypes: BRAZIL: São Paulo: Parque Estadual de Campos do Jordão, Rio Galharada, 22 $2^{\circ} 1^{\prime} 40 " S, 045^{\circ} 27^{\prime} 47^{\prime \prime} \mathrm{W}, 1530$ m, 13-15.ix.2002 (Blahnik, Prather, Melo, Huamantinco) 1 male (UMSP000200916) (UMSP), 1 female (UMSP000200917) (UMSP), 1 female (UMSP000200918) (MZUSP).

Etymology: The specific epithet, paulista, refers to the inhabitants of the Brazilian state of São Paulo, where this new species also resides.

\section{Additional Species Records}

We present below new distribution records for Notalina (Neonotalina) from Brazil to further document the distribution of the genus in the Neotropics.

\section{Notalina brasiliana Holzenthal 1986}

BRAZIL: Minas Gerais: Parque Estadual do Rio Preto, Rio Preto, $18^{\circ} 07^{\prime} 10^{\prime \prime} \mathrm{S}$, 04320'28"W, $830 \mathrm{~m}, 14 . x i .2001$ (Blahnik \& Amarante) 1 male, 2 females (UFRJ); Parque Estadual do Rio Preto, Córrego das Eguas, 1808'43"S, 043²2'09"W, $891 \mathrm{~m}$, 14.x.2000 (Paprocki, Amarante, \& Isaac) 5 males, 5 females (MZUSP); trib. to Rio Preto, 20 km (rd) S São Gonçalo do Rio Preto, 1806'53"S, 043²0'36"W, 700 m, 20.v. 1998 (Holzenthal \& Paprocki) 1 male, 2 females (NMNH); Rio Preto, 20 km (rd) S São

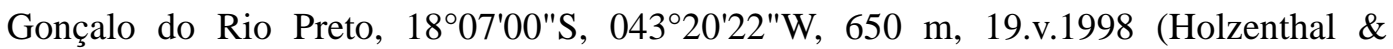
Paprocki) 1 female (MZUSP); trib. of Rio Parauninha, Serra do Breu, Fazenda do Zé da Mata, 1907'55"S, 04337'24"W, 15.ii.1999 (Paprocki \& Braga) 40 males, 22 females (UMSP).

\section{Notalina cipo Holzenthal 1986}

BRAZIL: Minas Gerais: Rio Caraça, near Santa Barbara, 2001'22"S, 043²8'45"W, 728 m, 9.xi.2001 (Holzenthal, Amarante, Blahnik, \& Paprocki) 1 male, 3 females (UMSP); Serra do Cipo, trib. to Rio Capivara, 19¹4'24"S, 043³4'56"W, 1000 m, 18.ii.1998 (Holzenthal \& Paprocki) 1 male, 2 females (MZUSP); Aldeia de Cachoeira das Pedras, 2006'49"S, 04401'25"W, 925 m, 28-29.ix.2000 (Paprocki \& Braga) 1 male (UFRJ); Córrego da Serra de Ouro Fino, Vale do Tropeiro, 20¹2'22"S, 043³8'35"W, 1000 m, 8.x.2000 (Paprocki, Salgado, \& Isaac) 1 male (NMNH).

\section{Notalina hamiltoni Holzenthal 1986}

BRAZIL: São Paulo: Parque Estadual de Campos do Jordão, Cachoeira Galharada, 22 ${ }^{\circ} 41^{\prime} 44^{\prime \prime} \mathrm{S}, 045^{\circ} 27^{\prime} 43^{\prime \prime} \mathrm{W}, 1620$ m, 15.x.1998 (Paprocki \& Froehlich) 1 male, 2 females 

16.ix.2002 (Blahnik, Prather, \& Huamantinco) 8 males, 1 female (UMSP), 2 males (UFRJ), 1 male (MZUSP), 1 male (NMNH).

\section{Notalina morsei Holzenthal 1986}

BRAZIL: Minas Gerais: Parque Estadual do Itacolomi, trib. to Rio Belchior, 20 25'18"S, 0432ㄴ'42"W, 700 m, 6.xi.2001 (Holzenthal, Amarante, Blahnik, \& Paprocki) 4 males (MZUSP); Corrego das Aguas Pretas \& tribs., ca. $15 \mathrm{~km} \mathrm{~S}$ Aiuruoca, 22 $03^{\prime} 42^{\prime \prime S}$, 044³8'14"W, 1386 m, 21.xi.2001 (Holzenthal, Blahnik, Neto, \& Paprocki) 2 males, 3 females (UMSP). Rio de Janeiro: Parque Nacional do Itatiaia, Rio Campo Belo, 2227'02"S, 044³6'49"W, 1300 m, 23.xi.2001 (Holzenthal, Blahnik, Neto, \& Paprocki) 2 males (UFRJ); 7.iii.2002 (Holzenthal, Blahnik, Paprocki, \& Prather) 2 males, 1 female (UMSP); Rio Campo Belo, trail to Veu da Noiva, 22 $2^{\circ} 5^{\prime} 42^{\prime \prime} \mathrm{S}, 044^{\circ} 37^{\prime} 10^{\prime \prime} \mathrm{W}, 1310 \mathrm{~m}$, 24.xi.2001 (Holzenthal, Blahnik, Neto, \& Paprocki) 5 males, 2 females (UMSP); Parque

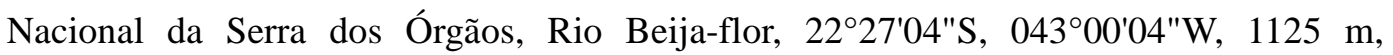
27.ii.2002 (Holzenthal, Blahnik, Paprocki, \& Prather) 3 males, 1 female (UFRJ); Rio Macaé, Macaé de Cima, 22²3'41"S, 042³0'08"W, 1000 m, 8.iii.2002 (Holzenthal, Blahnik, Paprocki, \& Prather) 5 males, 4 females (UMSP); Rio das Flores, Macaé de Cima, 10 km SE Mury, 1000 m, 9.iii.2002 (Holzenthal, Blahnik, Paprocki, \& Prather) 1 male (UMSP). São Paulo: Serra do Japi, Córrego Paraiso near main building, 23ํ'14'00"S, 046 56'12"W, 8.xii.1997 (Froehlich) 3 males, 6 females (MZUSP).

\section{Notalina spp. (undetermined females)}

BRAZIL: Minas Gerais: Rio Caraça, near Santa Barbara, 2001'22"S, 04328'45"W, 728 m, 9.xi.2001 (Holzenthal, Amarante, Blahnik, \& Paprocki) 1 female (UMSP); Parque

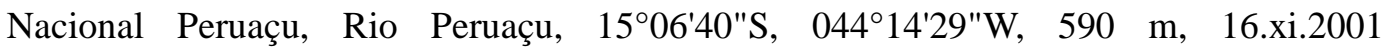
(Holzenthal, Amarante, Blahnik, \& Paprocki) 1 female (UMSP); Parque Estadual Itacolomi, Rio Belchior, 20²5'02"S, 0432 25'38"W, 725 m, 24.i.1999 (Amarante) 1 female (UMSP); Serra do Cipó, Capão da Mata, 19¹9'21"S, 043³2'15"W, 1170 m, 10.iii.1996 (Holzenthal, Rochetti, \& Oliveira) 1 female (UMSP); same, except 13-14.ii.1998 (Holzenthal \& Paprocki) 1 female (UMSP); Parque Estadual do Rio Preto, trib. to Rio Preto, 20 km (rd) S São Gonçalo do Rio Preto, 1806'53"S, 04320'36"W, 700 m, 14.xi.2001 (Holzenthal \& Paprocki) 5 females (UMSP); Estação Ecológica de Peti, Córrego Brucutu, 1953'00"S, 04322'27"W, 29.ix.1998 (Paprocki) 2 females (UMSP); Ibitipoca, Sitio of Anestis Papadopolous, 21 43'14"S, 04354'33"W, 1200 m, 23.x.2000 (Paprocki) 3 females (UMSP); Rio Parauninha, Serra do Breu, Fazenda do Zé da Mata, 19¹0'59"S, 04343'29"W, $14.1 i .1999$ (Paprocki \& Braga) 1 female (UMSP); Parque Nacional do Caparaó, Rio Caparaó, Vale Verde, $20^{\circ} 25^{\prime} 02^{\prime \prime} \mathrm{S}, 041^{\circ} 50^{\prime} 46^{\prime \prime} \mathrm{W}, 1350 \mathrm{~m}, 12$ 13.iii.2002 (Holzenthal, Blahnik, Paprocki, \& Prather) 1 female (UMSP). Paraná: trib. to 
Rio Mãe Catira, 10.5 km. N Porto de Cima, 25²1'47"S, 04852'35"W, 200 m, 10.xii.1997

(Holzenthal \& Huisman) 1 female (UMSP). Rio de Janeiro: Parque Nacional do Itatiaia,

Rio Campo Belo, trail to Veu da Noiva, 22 ${ }^{\circ} 5^{\prime} 42^{\prime \prime} \mathrm{S}, 044^{\circ} 37^{\prime} 10^{\prime \prime} \mathrm{W}, 1310 \mathrm{~m}$, 5.iii.2002 (Holzenthal, Blahnik, Paprocki, \& Prather) 2 females (UMSP); Rio Macaé, Macaé de Cima, 22 $23^{\circ} 41^{\prime \prime S}, 042^{\circ} 30^{\prime} 08^{\prime \prime} \mathrm{W}, 1000$ m, 8.iii.2002 (Holzenthal, Blahnik, Paprocki, \& Prather) 1 female (UMSP). São Paulo: Parque Estadual de Campos do Jordão, Cachoeira Galharada, 2241'44"S, 045²7'43"W, 1620 m, 7.iii.1996 (Holzenthal, Rochetti, \& Oliveira) 11 females (UMSP); Estação Biológica Boraceia, Rio Guaratuba, 2340'02"S, 04553'46"W, 775 m, 17.iv.1998 (Holzenthal, Melo, \& Froehlich) 1 female (UMSP); Parque Estadual de Campos do Jordão, Rio Galharada, 22 $41^{\prime} 40 " \mathrm{~S}, 045^{\circ} 27^{\prime} 47^{\prime \prime} \mathrm{W}, 1530 \mathrm{~m}$, 4-5.iii.1996 (Holzenthal \& Guahyba) 1 female (UMSP); same, except 22.i.1998 (Holzenthal, Froehlich, \& Paprocki) 1 female (UMSP); Estação Biológica Boraceia, Rio Venerando \& tribs., 2339'11"S, 04553'25"W, 850 m, 18-20 Apr 1998 (Holzenthal, Melo, \& Froehlich) 1 female (UMSP); Serra do Japi, Córrego da Ermida and small dam, $23^{\circ} 14^{\prime} \mathrm{S}$, 046 56'W, 9-10.xii.1997 (MZUSP) 1 female (MZUSP); Rio Casquilho, $3.4 \mathrm{~km}$ NE Parque Estadual Campos do Jordão, 2240'17"S, 045²7'52"W, 1550 m, 23.i.1998 (Holzenthal, Froehlich, \& Paprocki) 1 female (UMSP); Rio do Casquilho, Bosque Vermelho, ca. $5 \mathrm{~km}$ from Parque Estadual de Campos do Jordão, 2240'00"S, 045²7'30"W, $1435 \mathrm{~m}$, 16.ix.2002 (Blahnik, Prather, Melo, Huamantinco) 1 female (UMSP).

\section{Results}

Phylogenetic interpretations. The unweighted phylogenetic analysis yielded 14 equally parsimonious trees, with length (L) 69, consistency index (CI) 0.56 , retention index (RI) 0.80 , and rescaled consistency index (RC) 0.45 . The differences among these topologies, from the equally weighted analyses, occurred only within the outgroup taxa, especially among the species of Notalina (Notalina); relationships among the species of $N$. (Neonotalina) were constant. The same consensus was obtained from successive weighting analyses.

The consensus of implied weighting in the phylogenetic analysis with $\mathrm{k}$ values ranging from 1-3 yielded three most parsimonious solutions. The consensus showed 2 polytomies within Notalina. The consensus of the topologies obtained with $\mathrm{k}=4-15$ were the same and corresponded to one of the solutions of the analysis with $k=1-3$. The strict consensus tree is presented in Fig. 3.

The topology shows Notalina (Notalina) and Notalina (Neonotalina) as monophyletic, both based on a reliable set of unique characters. Because the focus of the analysis is on the relationships among $N$. (Neonotalina) species, the results at more basal positions in the tree should be interpreted with due care, because of undersampling of taxa and characters which may yield grouping based on homoplasies between ingroup and outgroup species. In this sense, four unique characters support the monophyly of Notalina. 
Within $N$. (Neonotalina) two separate clades are clear, the roraima group and the brasiliana group, and are supported by a number of uniquely derived characters. In the roraima group, $N$. nanay appears as the sister group of $N$. matthiasi $+N$. roraima. The monophyly of this pair of species is supported by a homoplastic character 2(0), present also in the brasiliana group.

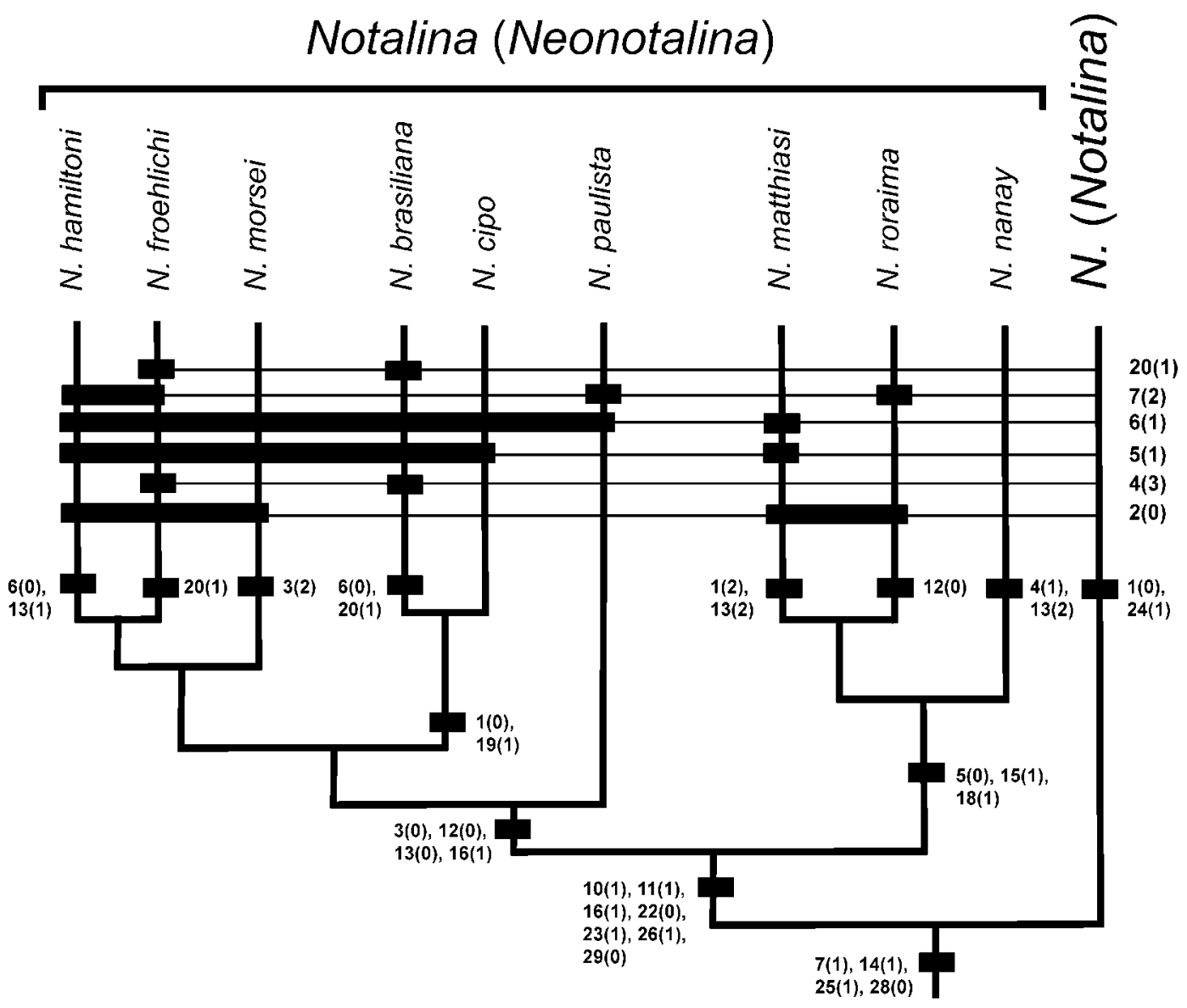

FIGURE 3. Strict consensus of 14 cladograms from equally weighted parsimony analysis, reflecting the phylogeny of Notalina (Neonotalina). Successive weighting and implied weighting (K values among 4 and 15) analyses yielded identical configuration. Characters of unique origin in the cladogram are placed at the nodes, while the distribution of characters of multiple origins is given at the top. Because the phylogenetic signal (i.e., sampled characters) is weak for relationships among outgroups, only the results for the analysis within Notalina (Neonotalina) has been considered.

In the brasiliana group, $N$. paulista appears as a sister to the set of remaining species of the group, but there is a single character of homoplastic origin 6(1) showing a heterobathmy of characters at this level. Within this smaller group, two clades are formed, one composed of $N$. brasiliana and $N$. cipo, based on two unique features1(0) and 19(1), 
while the other group is composed of $N$. morsei and $N$. froehlichi $+N$. hamiltoni, based on a single homoplastic feature 2(0). The monophyly of $N$. froehlichi $+N$. hamiltoni is shown by character $7(2)$, a feature also present in $N$. brasiliana and $N$. roraima. Two apomorphic features 4(3) and 20(1) are shared by $N$. froehlichi and N. brasiliana, which belong to different clades within the subgenus. However, the features associating $N$. brasiliana and $N$. cipo 6(0) and 19(1) have unique origins, while other features keep together $N$. froehlichi and $N$. hamiltoni, so similarities between $N$. froehlichi and $N$. brasiliana should be seen as artificial. Even though additional features would be helpful to corroborate the clades obtained here, the topology is considerably stable to allow biogeographical inferences.

Biogeographic considerations. Morse (1981) suggested evidence for the origin of the Leptoceridae at 65 million years ago (Ma), while Weaver (1984) accepted a Gondwanan origin for the family. Weaver's (1984) hypothesis by extension allows the interpretation of ancestral Triplectidinae occurring in southern Gondwana (South America and Australia, $65 \mathrm{Ma}$ ). The main geological event at this time was the separation of Africa from the supercontinent including South American-Antarctic-Australia. If this is correct, the division of southern Gondwanaland was responsible for the vicariance-induced disjunction within the Triplectidinae. Under this interpretation, the Leptoceridae would have to be already diversified in Gondwanaland, moving the origin of the family back to Pangea. This would explain basal groups of Leptoceridae in the northern hemisphere, in warmer as well as termperate (New Zealand) areas of Gondwanan affinities. This would take the origin of the Leptoceridae well back to $180 \mathrm{Ma}$. The records of Triplectides in Baltic amber (Eocene/Oligocene) (Ulmer 1912, Botosaneanu and Wichard 1981) do not contradict this hypothesis, even though fossil recent genera of Triplectidinae in Cretaceous amber would be expected. The 15 genera of Triplectidinae are distributed in South and Central America, Australia, New Zealand, and southeast and eastern Asia. The Asian distribution of Triplectides would be, in this case, due to dispersion from south to north, favoring Holzenthal's (1986a) view.

Three primary areas of endemism were proposed by Holzenthal (1985) for the Neotropical leptocerid fauna, two in the Brazilian subregion and the other in the Chilean subregion, comprising southern Patagonia (southern Chile and adjacent Argentina). One of the Brazilian subregion areas of endemism includes northern South America, extending to the tropical portion of Mexico, the other comprising the highlands of southeastern Brazil, with possible extension to northeast Argentina and Paraguay. Holzenthal (1986a) later proposed that the disjunct distribution and diversification of several Neotropical triplectidine genera could have been the result of Quaternary climatic and geological events, including Pleistocene glaciation, or the formation of Amazon Basin (Miocene/ Pliocene). This would also apply to the origin of the brasiliana and roraima speciesgroups of Notalina (Neonotalina).

Notalina (Neonotalina) is present in higher areas with colder climate in South America, namely, in southeastern Brazil (the brasiliana group) and in Brazilian- 

this does not solve the problem of historical association of the species or of the subgenus to tropical or temperate areas of the supercontinent. If the origin of the subgenus is associated with the tropical Gondwanaland, species of $N$. (Neonotalina) would be expected to be found in warmer parts of South America, as well as in Africa and South Asia. If its origin is associated with temperate Gondwanaland, this would link the clade to Chile, New Zealand and southeastern Australia, usually referred to as groups with circumAntarctic distribution. Even though groups with temperate origin are usually restricted to southern Brazil, e.g., the dipteran genera of Ditomyiidae, Nervijuncta (Freeman 1951, Munroe 1974), in some cases, they extend north along the Andes, as the other South American Ditomyiidae genus, Australosymmerus. Even though additional records may come to favor the hypothesis of a tropical connection of the subgenus, it seems more reliable that $N$. (Neonotalina) corresponds to a Neotropical clade of a circum-temperate taxon. This hypothesis places the origin of Notalina (Neonotalina) in the Lower Paleocene and the diversification of the group during the rest of the Cenozoic, which would explain some diversification within the species-groups brasiliana and roraima. By extension, this agrees with the interpretation of Holzenthal (1985) for the position of areas of endemism, but with a different assignment for the origin of clades occupying these areas.

\section{Acknowledgments}

We are grateful to Dr. R. Blahnik (University of Minnesota) for valuable assistance in determining the identity of the new species, to M. I. P. A. Balbi (Universidade de São Paulo) for support with specimen preparation and to Dr. S. Weller (University of Minnesota) for very useful suggestions on the analyses. We also thank Mr. H. Paprocki (Pontifícia Universidade Católica de Minas Gerais), Mr. C. M. D. Santos (Universidade de São Paulo), and Dr. C. Froehlich (Universidade de São Paulo) for their helpful comments on the manuscript. This work was supported by a CNPq fellowship (Conselho Nacional de Desenvolvimento Científico e Tecnológico do Brazil) to ARC and NSF Grants 9971885 and 0117772 to RWH.

\section{References}

Blahnik, R.J. \& Holzenthal, R.W. (2004) Collection and curation of Trichoptera, with an emphasis on pinned material. Nectopsyche, Neotropical Trichoptera Newsletter, 1, 8-20. Available from http://www.entomology.umn.edu/museum/links/news.html (accessed 15 September 2005).

Botosaneanu, L. \& Wichard, W. (1981) Trichoptera from Baltic Amber. In: Moretti, G.P. (Ed.), Proceedings of the 3rd International Symposium on Trichoptera. W. Junk Publishers. The Hague, pp. 31-32.

Brauer, F. (1865) Zweiter berichte über die auf der Weltfahrt der Kais. Fregatte Novara gesammelten Neuropteren.Verhandlungen der Kaiserlich-königlichen Zoologischen-Botanischen 
Gesellschaft in Wien, 15, 415-422.

Burmeister, H. (1839) Handbuch der Entomologie. Zweiter Band, Zweite Ubtheilung. Berlin, Theod. Chr. Friedr. Enslin, xii + 397-1050.

Carpenter, J.M. (1988) Choosing among equally parsimonious cladograms. Cladistics, 4, 291-296. Carpenter, J.M. (1994) Successive weighting, reliability and evidence. Cladistics, 10, 215-220.

Carpenter, J.M., Strassmann, J.E., Turillazzi, S., Hughes, C.R., Solis, C.R. \& Cervo, R. (1993) Phylogenetic relationships among paper wasp social parasites and their hosts (Hymenoptera: Vespidae; Polistinae). Cladistics, 9, 129-146.

Carpenter J.M., Kojima, J.I. \& Wenzel, J.W. (2000) Polybia, paraphyly, and polistine phylogeny. American Museum Novitates, 3298, 1-24.

Farris, J.S. (1969) A successive approximations approach to character weighting. Systematic Zoology, 18, 374-385.

Flint, O.S., Jr. (1983) Studies of Neotropical caddisflies, XXXIII: new species from austral South America (Trichoptera). Smithsonian Contributions to Zoology, 377, 1-100.

Flint, O.S., Jr., Holzenthal, R.W. \& Harris, S.C. (1999) Catalog of the Neotropical Caddisflies (Trichoptera). Special Publications, Ohio Biological Survey, Columbus, Ohio, 239 pp.

Freeman, P. (1951) Diptera of Patagonia and South Chile. Part III - Mycetophilidae. The British Museum (Natural History), London, 138 pp., plates I-XLIX.

Goloboff, P.A. (1993) Estimating character weights during tree search. Cladistics, 9, 83-91.

Goloboff, P.A. (1995) Parsimony and weighting: a reply to Turner and Zandee. Cladistics, 11, 91104.

Hendy, M.D. \& Penny, D. (1982) Branch and bound algorithms to determine minimal evolutionary trees. Mathematical Biosciences, 59, 277-290.

Hillis, D.M. (1991) Discriminating between phylogenetic signal and random noise in DNA sequences. In: Miyamoto, M.M. \& Cracraft, J. (Eds.). Phylogenetic Analysis of DNA Sequences, Oxford University Press, New York, 278-294.

Holzenthal, R.W. (1985) Studies in Neotropical Leptoceridae (Trichoptera) IV: a revision of Brachysetodes Schmid. Transactions of the American Entomological Society, 111, 407-440.

Holzenthal, R.W. (1986a) The Neotropical species of Notalina, a southern group of long-horned caddisflies (Trichoptera: Leptoceridae). Systematic Entomology, 11, 61-73.

Holzenthal, R.W. (1986b) Studies in Neotropical Leptoceridae (Trichoptera), VI: Immature stages of Hudsonema flaminii (Navás) and the evolution and historical biogeography of Hudsonemini (Triplectidini). Proceedings of the Entomological Society of Washington, 88, 268-279.

Holzenthal, R.W. (1988) Studies in Neotropical Leptoceridae (Trichoptera), VIII: The Genera Atanatolica Mosely and Grumichella Müller (Triplectidinae: Grumichellini). Transations of the America Entomological Society, 114, 71-128.

Holzenthal, R.W. \& Andersen, T. (2004) The caddisfly genus Triaenodes in the Neotropics (Trichoptera: Leptoceridae). Zootaxa, 511, 1-80.

Kluge, A.G. (1997). Testability and the refutation and corroboration of cladistic hypotheses. Cladistics, 13, 81-96.

Leach, W.E. (1815) Entomology. In: Sir Brewster, D. (Ed.), The Edinburgh Encyclopedia. William Blackwood, Edinburgh, 9, 57-172.

Moor, F.C. (1997) An unusual caddisfly larva from South Africa, a possible member of the Triplectidinae (Trichoptera: Leptoceridae). In: Holzenthal, R.W. \& Flint, O.S., Jr. (Eds.), Proceedings of the 8th International Symposium on Trichoptera. Ohio Biological Survey, Columbus, pp. 323-330.

Morse, J.C. (1981) A phylogeny and classification of family group taxa of Leptoceridae (Trichoptera). In: Moretti, G.P (Ed.), Proceedings of the 3rd International Symposium on Trichoptera, W. Junk Publishers, The Hague, Series Entomologica 20, pp. 257-264.

Morse, J.C. \& Holzenthal, R.W. (1987) Higher classification of Triplectidinae (Trichoptera: Lepto- 
ceridae). In: Bournaud, M. \& Tachet, H. (Eds.), Proceedings of the 5th International Symposium on Trichoptera, W. Junk Publishers, Dordrecht, The Netherlands, Serie Entomologica 39, pp. 139-144.

Morse, J.C. (2003) Trichoptera (Caddisflies). In: Resh, V.H. \& Cardé, R.T. (Eds.), Encyclopedia of Insects. Academic Press, San Diego, California, pp. 1145-1151.

Mosely, M.E. (1936) A revision of the Triplectidinae, a subfamily of the Leptoceridae (Trichoptera). Transactions of the Royal Entomological Society of London, 85, 91-130.

Mosely, M.E. \& Kimmins, D.E. (1953) The Trichoptera (Caddis-flies) of Australia and New Zealand. London, British Museum (Natural History), $550 \mathrm{pp}$.

Munroe, D.D. (1974) The systematics, phylogeny, and zoogeography of Symmerus Walker and Australosymmerus Freeman (Diptera: Mycetophilidae: Ditomyiidae). Memoirs of the Entomological Society of Canada, 92, 1-183.

Navás, R.P.L. (1926) Insectos Neotropicos (2a serie). Revista Chilena de Historia Natural, 30, 326-336.

Neboiss, A. (1977) A taxonomic and zoogeographic study of Tasmanian caddis-flies. Memoirs of the National Museum of Victoria, 38, 1-208, pls. 1-3.

Neboiss, A. (1982) The caddis-flies (Trichoptera) of south-western Australia. Australian Journal of Zoology, 30, 271-325.

Neboiss, A. (1984) Four new caddis-fly species from Victoria (Trichoptera: Insecta). The Victorian Naturalist (Melbourne), 101, 86-91.

Neboiss, A. (1986) Atlas of Trichoptera of the SW Pacific-Australian Region. W. Junk Publishers, Dordrecht, Series Entomologica, 37, $286 \mathrm{pp}$.

Nixon, K.C. \& Carpenter, J.M. (1993) On Outgroups. Cladistics, 9, 413-426.

Page, R. (2001) Nexus Data Editor for Windows (NDE), Version 0.5.0. Program and Documentation.

Paprocki, H., Holzenthal, R.W. \& Blahnik, R.J. (2004) Checklist of the Trichoptera (Insecta) of Brazil I. Biota Neotropica, 4, 1-22.

Schmid, F. (1980) Genera des Trichoptères de Canada et des États adjacents. Les Insectes et Arachnides du Canada, part 7. Agriculture Canada, Publication 1692. Ottawa. 296 pp.

St. Clair, R. (1991) The genus Notalina (Trichoptera: Leptoceridae: Triplectidinae) in southeastern Australia, with descriptions of the larvae and pupae. Invertebrate Taxonomy, 4, 895-934.

Swofford, D.L. (2001) PAUP*: Phylogenetic Analysis Using Parsimony (*and other Methods), ver. 4.0b10, Sinauer, Sunderland, Massachusetts.

Ulmer, G. (1906) Neuer Beitrag zur Kenntnis aussereuropaeischer Trichopteren. Notes from the Leyden Museum, 28, 1-116.

Ulmer, G. (1907) Trichoptera. In: Wytsman, P. (Ed.), Genera Insectorum, 60a, 1-259, $41 \mathrm{pl}$.

Ulmer, G. (1912) Die Trichopteren des Baltischen Bernsteins. Beiträge zur Naturkundlichen Preussen, 10, 1-380.

Weaver, J.S., III. (1984) The evolution and classification of Trichoptera, Part I: The Groundplan of Trichoptera. In: Morse, J.C. (Ed.), Proceedings of the 4th International Symposium on Trichoptera. W. Junk Publishers, The Hague, The Netherlands, pp. 413-419. 